\title{
Alendronate is best for prevention of bone loss
}

Alendronate is superior to alfacalcidol for prevention of glucocorticoid-associated bone loss in patients with ulcerative colitis, report Mitsuyama and colleagues.

Bone loss is common in patients with ulcerative colitis on glucocorticoid therapy and leads to increased risk of fractures and associated morbidity. Dr Mitsuyama, who performed this study with a group from Kurume University School of Medicine, Japan, says they are the "...first to compare the efficacy of bisphosphonate[s] [alendronate] with active vitamin $\mathrm{D}_{3}$ [alfacalcidol] for the treatment of this condition."

The team investigated the effects of these two drugs in 39 patients with ulcerative colitis who were randomly allocated to receive alendronate ( $5 \mathrm{mg}$ daily) or alfacalcidol ( $1 \mu \mathrm{g}$ daily) for 12 months. Bone loss was assessed by bone mineral density, bone formation by serum levels of bone alkaline phosphatase and bone resorption by urinary levels of $N$-telopeptide of type 1 collagen. Only alendronate was associated with significantly increased bone mineral density in the lumbar spine and significantly decreased serum levels of bone alkaline phosphatase. In addition, urinary levels of $N$-telopeptide of type 1 collagen levels were significantly decreased in the alendronate group. The number and type of adverse events were similar between groups.

Mitsuyama calls for "...these promising findings to be validated in large comparative trials." The researchers conclude that patients with ulcerative colitis on glucocorticoids should receive bisphosphonates rather than active vitamin $\mathrm{D}_{3}$.

\section{Rachel Jones}

Original article Kitazaki, S. et al. Clinical trial: comparison of alendronate and alfacalidol in glucocorticoid-associated osteoporosis in patients with ulcerative colitis. Aliment. Pharmacol. Ther. 29, 424-430 (2009). 